\title{
An Investigation into the Use of Tactons to Present Progress Information
}

\author{
Stephen Brewster and Alison King \\ Glasgow Interactive Systems Group, Department of Computing Science, \\ University of Glasgow, Glasgow, G12 8QQ, UK \\ stephen@dcs.gla.ac.uk \\ www.dcs.gla.ac.uk/ stephen
}

\begin{abstract}
This paper presents an initial investigation into the use of Tactons, or tactile icons, to present progress information in desktop human-computer interfaces. Progress bars are very common in a wide range of interfaces but have problems. For example, they must compete for screen space and visual attention with other visual tasks such as document editing or web browsing. To address these problems we created a tactile progress indicator, encoding progress information into a series of vibrotactile cues. An experiment comparing the tactile progress indicator to a standard visual one showed a significant improvement in performance and an overall preference for the tactile display. These results suggest that a tactile display is a good way to present such information and this has many potential applications from computer desktops to mobile telephones.
\end{abstract}

\section{Introduction}

Progress bars are a common feature of most graphical human-computer interfaces. They are used to indicate the current state of a task which does not complete instantaneously, such as downloading documents from the web or copying files. Myers [14] showed that people prefer systems with progress indicators, as they give novices confidence that a task is progressing successfully, whilst experts can get sufficient information to predict the approximate completion time of the task.

The problem with visual progress bars is that they can become hidden behind other windows on the desktop and often have to compete for visual attention with other tasks the user is trying to perform. Tactile presentation has the potential to solve these problems: progress indicators are temporal and temporal patterns are well perceived through the skin. This paper presents an initial experimental investigation into a vibrotactile progress indicator that does not require visual attention, communicating the progress of a task via a series of tactile pulses.

\section{Previous Work}

For a progress bar to be effective at keeping the user informed about the state of the task, Conn [6] says that it should have good time affordance, i.e. the user must be able 
to tell "when things are okay and when there are problems, and can generally predict when a task will be completed". To do this, Conn suggests a progress bar should give an indication of eight task properties:

1. Acceptance: What the task is and whether it has been accepted.

2. Scope: The overall size of the task and the corresponding time it is expected to take;

3. Initiation: Clear indication that the task has successfully started;

4. Progress: Clear indication of the task being carried out, and the rate at which the overall task is approaching completion;

5. Heartbeat: Indication that the task is still "alive";

6. Exception: Indication that a task has errors;

7. Remainder: Indication of how much of the task remains and/or how much time is left before completion;

8. Completion: Clear indication of termination of the task and the status at termination.

Several types of progress indicators are commonly used, from 'egg-timer' or 'clock hands' cursors to progress bars (see Figure 1). This paper will consider the latter as they provide more information to the user about the task in progress. They are used when files are copied, transferred or downloaded, etc., and are very common in desktop computer interfaces. They also occur on devices such as mobile telephones or MP3 players, where progress bars are used to indicate the download of web pages or the transfer of photographs or sound files.

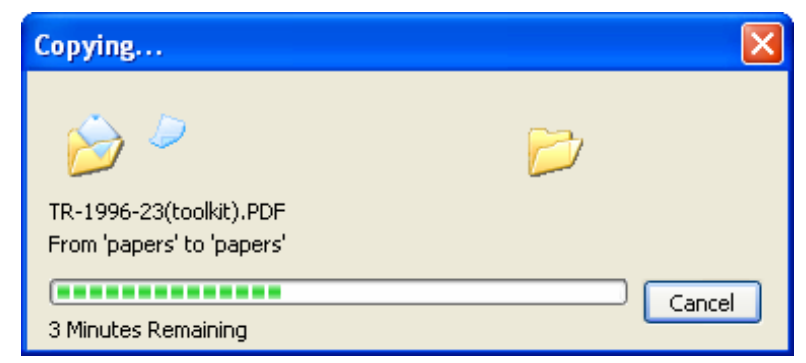

Fig. 1. The progress bar used by Microsoft Windows XP (www.microsoft.com/windowsxp)

Figure 1 shows a progress bar from the Windows XP operating system. In terms of Conn's properties the progress window itself and the type of task indicated in its title bar show Acceptance. Scope is given by the time remaining indicator under the progress bar. Initiation is indicated by the paper icon above the progress bar beginning to fly from the folder on the left to the one on the right. The progress bar itself gives and indication of the Progress of the task. The flying paper icon gives Heartbeat information. Exceptions will be indicated by an error window popping up over the progress bar. Remainder is indicated by the amount left on the progress bar and the time indicator. Completion is indicated by the disappearance of the progress window. 
The indicator presents information about progress very successfully, but there is one problem: users often move progress indicators to the edge of their displays, or cover them up with other windows so that they can get on with other tasks whilst, for example, files copy. This means that the display of information is lost. Users may occasionally bring the progress window to the front to see how things are going, but for much of the time it will be hidden. The problem is that the screen is a limited resource (even with large displays) and users want to maximize the amount they devote to their main tasks. A visual progress indicator must compete for visual attention with a primary task (e.g. typing a report) so the user ends up trying to concentrate on two visual tasks at once. In this paper we suggest that sharing the tasks between two different senses may be a better way to present this information; the user can look at the main task and feel the progress indicator.

\subsection{Audio Progress Indicators}

There has been some work into the design of sonic progress indicators that give information about progress using non-speech sounds, avoiding problems of screen space. Gaver [10] used the sound of liquid pouring from one container to another to indicate copying in his SonicFinder. The change in pitch of the sound gave the listener information about the how the copy was progressing and how close it was to the end. Crease and Brewster [7, 8] looked at using structured non-speech sounds called Earcons to indicate progress. They designed a system that presented Initiation, Progress, Heartbeat, Remainder and Completion. They used a low-pitched sound to represent the end of the progress task and a 'progress' sound to indicate the current amount of the task completed. This started at a high pitch and gradually lowered until it reached the pitch of the end sound. The listener knew when a task had completed because the two played at the same pitch. The design of our tactile progress indicator was partly based on this, but mapped into the time, rather than frequency, domain.

\subsection{Tactile Human-Computer Interaction}

There have been some good examples of the use of tactile displays to improve human-computer interfaces. Mackenzie and others have successfully shown that basic tactile feedback can improve pointing and steering type interactions $[1,5]$. Tactile cues can aid users in hitting targets such as buttons faster and more accurately. Lee $e t$ al. [13] and have recently developed a tactile stylus to use on touch screens and PDA's. Poupyrev et al. and Fukumoto et al. [9, 15, 16] have looked at the use of a tactile displays on handheld computers. Much of the focus of work in this area is on device and hardware development; until recently there were few tactile transducers routinely available and they were often designed for use in different domains (for example, sensory substitution systems [12]). Now many mobile telephones and PDAs have vibrotactile actuators included for alerting. These can be used for other purposes. Poupyrev et al. [16] have begun to look at interactions using the devices they have created. They describe a tactile progress bar where progress is mapped to the time between two clicks. They say it "... was easy to relate the tactile feedback to the current 
status of the process", but very little information is given on the design and no evaluation of its effectiveness is reported.

Techniques for encoding information in tactile cues have been investigated in the area of speech presentation to people with hearing impairments. Summers [17] used temporal patterns along with frequency and amplitude to encode speech information in vibrations, and found that participants mainly used information obtained from the temporal patterns, rather than from frequency/amplitude modulations. This suggests that rhythmic patterns would be a good place to start when designing cues for tactile displays.

Brewster and Brown have proposed Tactons, or tactile icons. These are structured, abstract messages that can be used to communicate tactually [2-4]. Information is encoded into Tactons using the basic parameters of cutaneous perception, such as waveform, rhythmic patterns and spatial location on the body. Their early results have shown that information can be encoded effectively in this way. Simple Tactons will be used in our system to indicate the state of progress.

\subsection{Audio Versus Tactile Presentation}

One disadvantage with the auditory display of progress is that either the user must wear headphones or use loudspeakers. Headphones tie the user to the desk and are not always appropriate, and loudspeaker presentation can be annoying to others nearby if the volume is up too high. The advantage of audio is that output devices are common and cheap and users can hear the display from anywhere around them.

Tactile displays do not have the issue with being public as they make no sound, so information can be delivered discretely. The disadvantage is that they must be in good contact with the skin for information to be perceived. Vibrotactile transducers are also not yet common on most desktop computers. If body location is to be used as a design parameter then transducers need to be mounted on different parts of the body and this can be intrusive. Mice such as the Logitech iFeel mouse (www.logitech.com) or most mobile phones and PDA's have a simple vibrotactile transducer built in. The problem is that if the user's hand is not on the mouse or phone then feedback will be missed.

One other issue is distraction. Carefully designed sounds can be habituated and fade into the background of consciousness, only coming to your attention when something changes (just as the sound of an air conditioner only gets your attention when it switches on or off, the rest of the time it fades into the background). It is not clear how we can design tactile displays to facilitate habituation. We easily habituate tactile stimuli (think of clothes for example) but it is not yet clear how we might design dynamic cues that do not annoy the user. We also, of course, need to avoid numbness by too much stimulation.

The choice of vibrotactile, auditory or visual display of information depends on how and when it will be used. At different times one or the other (or a combination of all three) might be most effective. Detailed study of interactions using tactile is needed to understand how to design them and when they should best be used. 


\section{Experiment}

An experiment was conducted to investigate if progress information could be presented using simple Tactons, and if presenting it this way would be more effective than its standard visual form.

\subsection{Design of a Tactile Progress Indicator}

The basic design of our progress indicator mapped the amount remaining of a download to the time between two pulses; the closer together the pulses the closer to the end of the download. The download is complete when the cues overlap. The time gap between the pulses is scaled to the amount being downloaded (up to a maximum of a 10 second gap in this case).

An Oboe timbre was used as the waveform for all of the cues. This gave a strong signal when presented through the transducer used. The Tactons were all played at a frequency of $250 \mathrm{~Hz}$; this is the resonant frequency of the transducer and also the optimum frequency of perception on the skin. The design of the progress indicator used three simple Tactons (the structure of the Tactons used is shown in Figure 2):

- Start: this indicated the start of a new download. A tone that increased in amplitude from 0 to maximum over a period of 1.5 seconds followed by 0.5 seconds at maximum amplitude was used.

- Current: this marked the current position of the progress indicator and was a single pulse lasting 0.5 seconds. For a new download this was played directly after the Start cue finished. Figure 3 shows the waveform of this stimulus.

- Target: this represented the end of the task. As the download progressed the Current stimuli got closer in time to the Target. When they overlapped the download was finished. The Target cue was a series of 4 short pulses, each lasting 0.6 seconds with a total length of 2.5 seconds. This made the two stimuli feel very different to avoid confusion.

According to Conn's properties this progress indicator gives information on Initiation (Start cue), Progress (movement of Current cue towards Target), Heartbeat (the pulsing of the Current cue), Remainder (the difference in time between the Current and Target cues), Completion (the combined Current and Target cue). Information was not given on Acceptance, in this case the task was always the same: file downloading. No Exceptions occurred in this experimental study so no feedback was needed.

A single VBW32 transducer was used (see Figure 4). This transducer was designed for use in tactile hearing aids, and is relatively low cost at US\$80. It was mounted on the top of the wrist of the non-dominant hand, under a sweat band to keep it tight against the skin. This kept it out of the way so that it did not interfere with typing. Headphones were worn (but not connected) to stop any sounds from the transducer being heard by the participant. The transducer is simple to use as it plugs into the headphone socket of a PC and is controlled by playing sound files. The use of a single transducer meant that this simple design could be used in a range of different devices, for example on a mobile telephone held in a user's hand. 


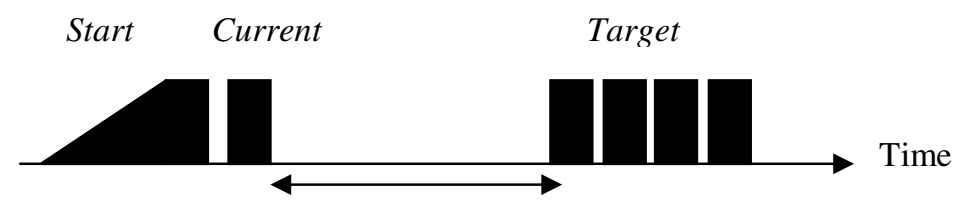

This gap is proportional to the amount of download remaining

Fig. 2. A schematic layout of the feedback used in the progress indicator for a new download. This would repeat (without the Start Tacton) until the download had completed

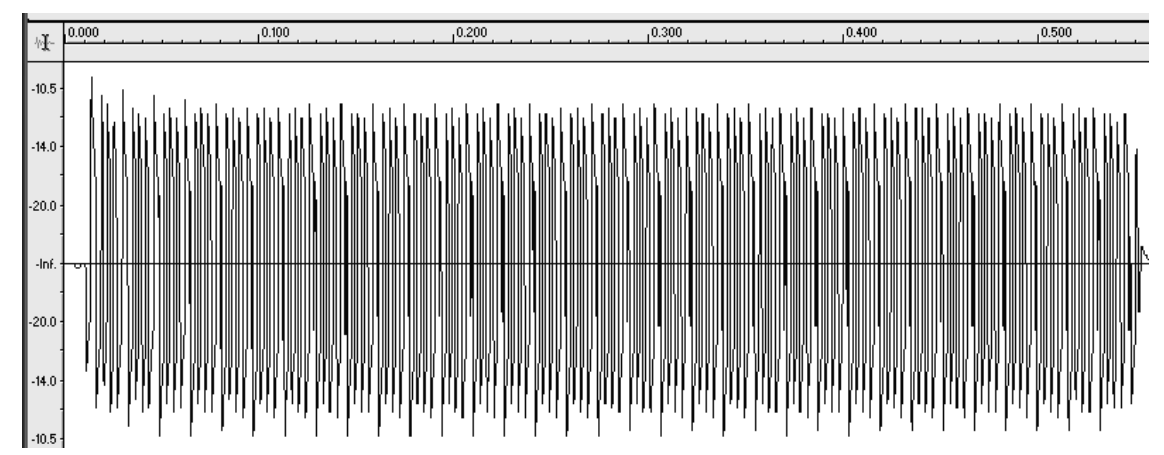

Fig. 3. Waveform of the Current Tacton



Fig. 4. The Tactaid VBW32 tactile transducer from Audiological Engineering Corporation (www.tactaid.com)

\subsection{Experimental Design and Procedure}

The experiment was a two-condition within subjects design. The independent variable was interface type with two levels: the standard visual progress bar and the tactile 
progress bar (with no visual display of progress). Participants experienced both interfaces with the order of presentation counterbalanced. The dependent variables were time to respond to the end of a download (the difference in time from when the download actually finished to when the user clicked the Finished button) and subjective workload.

Hart and Staveland [11] break workload into six different factors: mental demand, physical demand, time pressure, effort expended, performance level achieved and frustration experienced. NASA has developed a measurement tool, the NASA-Task Load Index (TLX) for estimating these subjective factors. We used this but added a seventh factor: Annoyance. In the experiment described here annoyance due to the tactile feedback was measured directly to find out if it was an issue. We also asked participants to indicate overall preference for the two interfaces.

The main experimental hypotheses were that the time taken to respond to the tactile stimuli would be shorter than for the visual stimuli. In addition, subjective workload would be significantly reduced by the inclusion of the tactile stimuli.

Fourteen subjects were used, all students from the University of Glasgow. Four reported themselves as touch-typists; the remainder as 'hunt-and-peck' typists.

The experimental task simulated a typical desktop interaction where the user had to type text and monitor file downloads at the same time. Participants typed in poetry which was given to them on sheets by the side of the computer used in the study. Their task was to type as much poetry as possible in the time of the experiment. Whilst typing they also had to monitor the download of a series of files and begin the download of the next as soon as the current one had finished.

The experimental software was run on a Windows XP machine with a 21 inch monitor set to a resolution of $1600 \times 1200$ pixels and the application maximized to full screen. Five downloads took place in each condition. These were the same for both conditions and ranged in time from 12 seconds to 1 minute. Two sets of poems were used, taken from the same source.

The Visual condition used a standard Microsoft Windows style progress bar, presented in the right hand corner of the screen (see Figure 5). On the left hand side of the screen was a large area for typing text. The Finished button was pressed when the participant noticed that a download had completed; when pressed it started the next download and recorded time to respond. (The Start button was used to start a condition and the Close button was used to close the application after the last download had been completed.)

The Tactile condition was exactly the same, except that the visual progress bar was not presented. The tactile cues described above were used to present the progress information in this condition.

Subjects were given a brief (approximately 5 minutes) training period before each condition. This gave them some training in the task they were about to perform and the cues they would receive. They received three practice downloads. After each condition they filled in NASA TLX workload charts. 


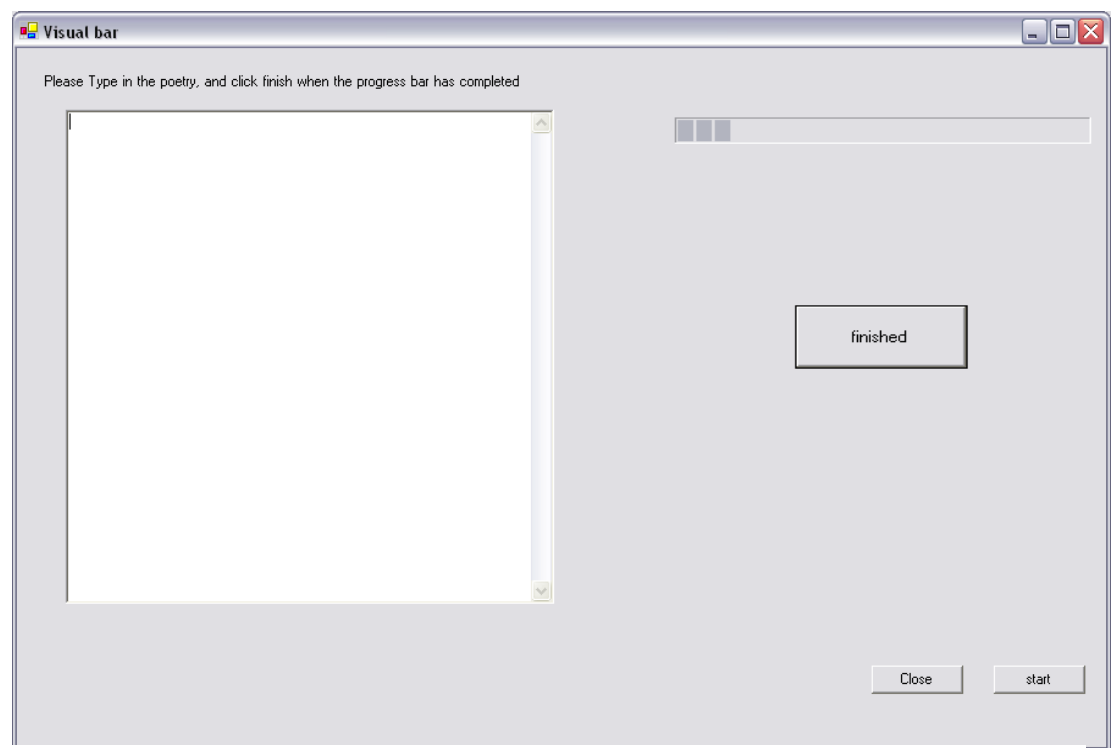

Fig. 5. The experimental interface for the Visual condition of the experiment

\subsection{Results}

The response times to the downloads are shown in Figure 5. The results show that the participants performed slower in the Visual condition with a mean time to respond of 13.54 seconds (SD 5.2) versus 8.7 seconds (SD 5.6) in the Tactile condition. A T-test showed a significant effect for interface type $\left(\mathrm{T}_{13}=3.23, \mathrm{p}=0.007\right)$, showing that participants noticed the end of a download significantly more quickly in the Tactile condition, confirming the first hypothesis.

In addition, the number of times the participants pressed the Finished button before the current download had finished was counted (this gives some idea of how well users understood the progress cues given). Participants clicked too early 4 out of 70 times in the Visual condition and 8 times in the Tactile. This suggests that users were monitoring well in both conditions, further confirmation that participants could use the tactile progress bar.

The results for subjective workload are presented in Figure 6. Overall workload (computed from the standard six workload factors) showed no significant difference between the two conditions with a mean of 8.5 (SD 2.4) for the Visual condition and 7.5 (SD 2.4) for the Tactile $\left(\mathrm{T}_{13}=0.88, \mathrm{p}=0.39\right)$. The second hypothesis was therefore not confirmed.

Annoyance showed no significant difference between conditions $\left(\mathrm{T}_{13}=1.38\right.$, $\mathrm{p}=0.19)$. Overall preference did show an effect with the Tactile condition significantly preferred over the Visual $\left(\mathrm{T}_{13}=4.00, \mathrm{p}=0.001\right)$. 


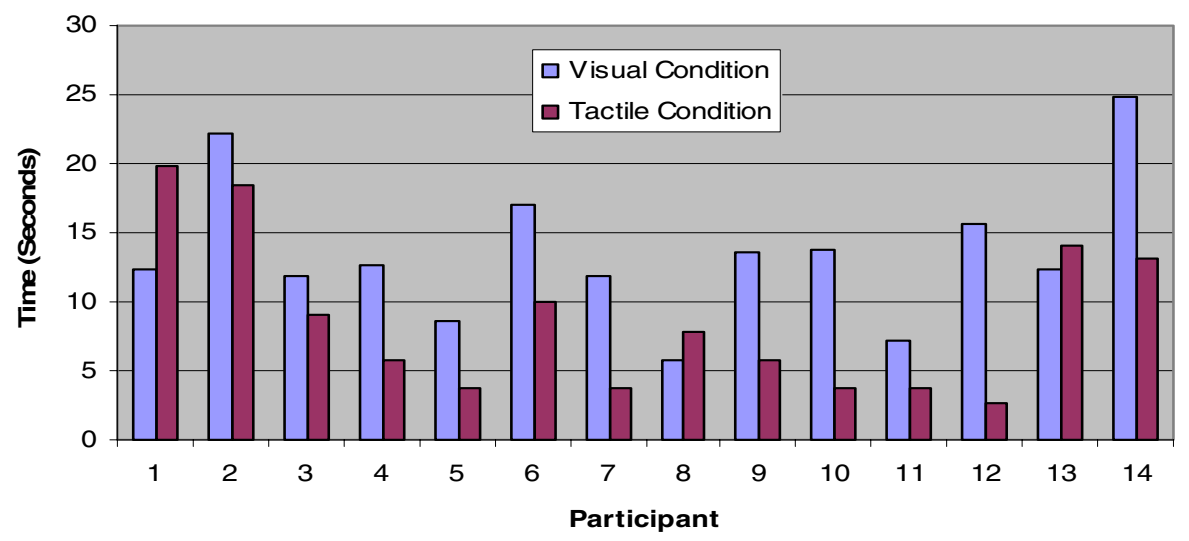

Fig. 6. Mean times to respond to the end of downloads

\subsection{Discussion and Future Work}

The results of this experiment showed that a simple tactile display could make a successful progress indicator. Participants responded more quickly to the tactile progress indicator than to the visual one. We suggest that this is because the use of the tactile display allowed participants to concentrate visual attention on their primary typing task whilst monitoring the background task of downloading files with their sense of touch, facilitating a sharing of the tasks between senses.

Workload was not significantly reduced by the tactile progress indicator, as predicted. Workload was improved in all categories apart from the mental demand of using the tactile progress indicator. This result may have been due to the unusual task; it is not common to monitor information presented in this way. The effect may be reduced with further exposure to such progress presentation. Participants did prefer the tactile display, which is positive, but this result should be taken with care as there could be some novelty effect. A longer term study would be needed to measure preference over time, but initial results are encouraging. In addition, a further study could look at performance in the typing task to see if users slowed down more or made more typing errors with one type of presentation or another.

Participants took a long time to respond to the end of downloads in both conditions. In Crease's experiment [8] participants responded in 5.3 seconds on average in the visual progress bar condition and 2.8 seconds in the audio. Part of the reason for the difference between this experiment and ours may have been the experimental instructions; in our experiment we told participants that the typing task was their main focus and that they should monitor downloads in the background. Another issue could have been the poetry used. This generally had short lines and it may have been that participants wanted to finish a line before responding to the progress bar (this appeared to happen in informal observations of some users). Therefore the absolute values of response times are less useful than the fact that there was a significant reduction in the Tactile condition. Crease's auditory progress indicator caused a $47 \%$ reduction in time to respond. Our tactile progress indicator 
caused a $36 \%$ reduction in time to respond. An interesting study would be to examine all three types of progress displays in one experiment to compare their performance.

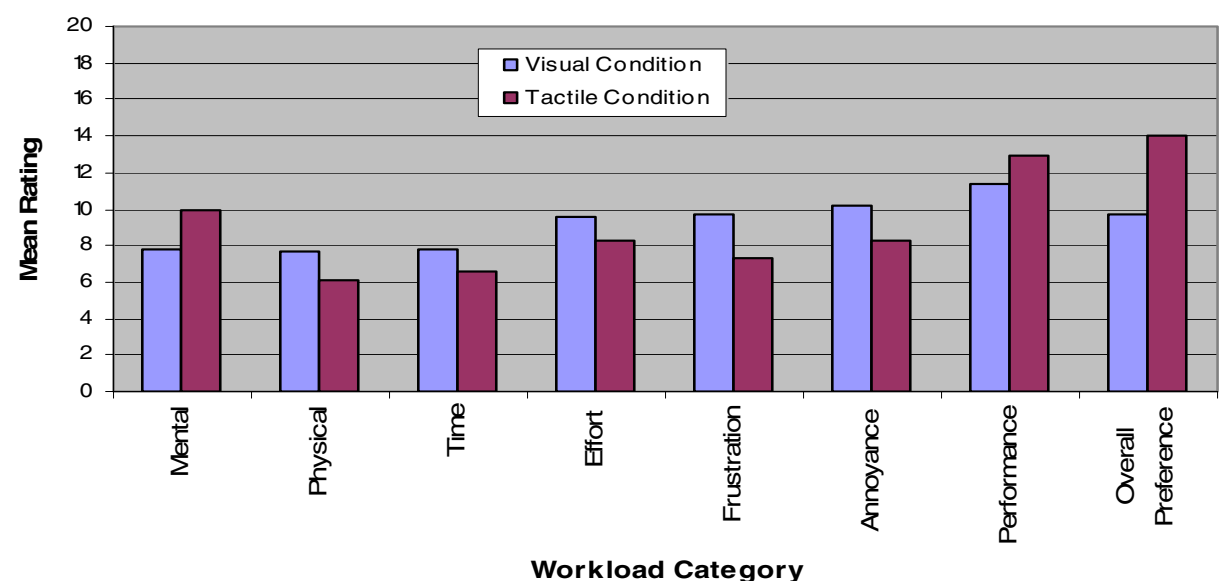

Fig. 7. Mean subjective workload results. Lower scores mean lower workload, except for Performance and Overall Preference where higher scores indicate better performance.

The design we created was simple, using just one transducer. This is beneficial as the cost of adding our tactile display is low so that such a progress indicator could be used in many different situations. Many mobile phones and handheld computers already have a basic tactile transducer in them for alerting purposes. We could use this to present progress information non-visually. This is particularly important as these devices have very limited screen space.

Further work should investigate other designs for the Tactons to see if we can get a faster response from users, for example. These were a first attempt and there is little useful guidance in the literature to facilitate good design. Since this experiment was performed Brown et al. [4] have begun to develop some design guidelines for Tactons and these could be incorporated into a future version. We could also make more sophisticated displays of progress information using multiple transducers. For example, a belt of transducers around the waist could be used. In this case a download might start at the front and then move around the body clockwise. When vibration is at the right hip $25 \%$ of a download is completed, when at the left hip $75 \%$, and $100 \%$ when the vibration reaches the front again. We will need to investigate if this gives a better perception of progress than the simple design presented here.

We have only looked at five of Conn's properties of progress indicators. A further step would be to design cues to represent the others. Acceptance might be difficult to present as some form of text is really needed to indicate what type of task has started, unless the possible set of different tasks is small. If that is the case then a Tacton could be included before the progress indicator starts to show its type. Exception would be easier as an error Tacton could be created that felt very different to the others to indicate problems and attract the user's attention. Scope might also be challenging, especially if the download is very large, as just leaving very long gaps 
between the tactile cues to show size is likely to confuse users because they will not know if the download has stopped or not. A Scope Tacton could be created that gave some indication of the overall size (perhaps a short Tacton for 'short' downloads, up to a longer one to represent 'long' downloads) and this could then be played before the main download started.

\section{Conclusions}

The experiment reported in this paper has shown that progress indicators can be presented in a tactile form, and that they can be more effective than standard visual progress bars. This is important as it allows users to keep their visual attention on a main task, such as typing, and use their sense of touch to receive information on the state of downloads. This experiment is one of the few that have investigated the design of tactile interactions. Much work is going into the development of new devices and hardware but less into the design of interactions using tactile displays. Our results show that it is possible to create effective desktop interactions using Tactons and further studies are planned to investigate other interactions. The simple design of our progress indicator also means that it may be applicable in other situations, for example handheld computers and mobile telephones could use such an indicator without sacrificing any valuable screen space.

\section{Acknowledgements}

This work was funded by EPSRC Advanced Research Fellowship GR/S53244.

\section{References}

1. Akamatsu, M., MacKenzie, I.S. and Hasbrouq, T. A comparison of tactile, auditory, and visual feedback in a pointing task using a mouse-type device. Ergonomics, 38. 816-827.

2. Brewster, S.A. and Brown, L.M., Non-Visual Information Display Using Tactons. In Extended Abstracts of ACM CHI 2004, (Vienna, Austria, 2004), ACM Press, 787-788.

3. Brewster, S.A. and Brown, L.M., Tactons: Structured Tactile Messages for Non-Visual Information Display. In Proceedings of Australasian User Interface Conference 2004, (Dunedin, New Zealand, 2004), Austalian Computer Society, 15-23.

4. Brown, L., Brewster, S.A. and Purchase, H., A First Investigation into the Effectiveness of Tactons. In Proceedings of World Haptics 2005, (Pisa, Italy, 2005), IEEE Press.

5. Campbell, C., Zhai, S., May, K. and Maglio, P., What You Feel Must Be What You See: Adding Tactile Feedback to the Trackpoint. In Proceedings of IFIP INTERACT'99, (Edinburgh, UK, 1999), IOS Press, 383-390.

6. Conn, A.P., Time Affordances: The Time Factor in Diagnostic Usability Heuristics. In Proceedings of ACM CHI'95, (Denver, Colorado, USA, 1995), ACM Press AddisonWesley, 186-193.

7. Crease, M. and Brewster, S.A., Scope for Progress - Monitoring Background Tasks with Sound. In Volume II of the Proceedings of INTERACT '99, (Edinburgh, UK, 1999), British Computer Society, 19-20. 
8. Crease, M.C. and Brewster, S.A., Making progress with sounds - The design and evaluation of an audio progress bar. In Proceedings of ICAD'98, (Glasgow, UK, 1998), British Computer Society.

9. Fukumoto, M. and Toshaki, S., ActiveClick: Tacile Feedback for Touch Panels. in Extended Abstracts of CHI 2001, (Seattle, WA, USA, 2001), ACM Press, 121-122.

10. Gaver, W. The SonicFinder: An interface that uses auditory icons. Human Computer Interaction, 4 (1). 67-94.

11. Hart, S. and Staveland, L. Development of NASA-TLX (Task Load Index): Results of empirical and theoretical research. in Hancock, P. and Meshkati, N. eds. Human mental workload, North Holland B.V., Amsterdam, 1988, 139-183.

12. Kaczmarek, K., Webster, J., Bach-y-Rita, P. and Tompkins, W. Electrotacile and vibrotactile displays for sensory substitution systems. IEEE Transaction on Biomedical Engineering, 38 (1). 1-16.

13. Lee, J.C., Dietz, P., Leigh, D., Yerazunis, W. and Hudson, S.E., Haptic Pen: A Tactile Feedback Stylus for Touch Screens. In Proceedings of UIST 2004, (Santa Fe, NM, USA, 2004), ACM Press Addison-Wesley, 291-294.

14. Myers, B.A., The Importance Of Percent-Done Progress Indicators for Computer-Human Interfaces. In Proceedings of ACM CHI'85, (San Fransisco, CA, USA, 1985), ACM Press Addison-Wesley, 11-17.

15. Poupyrev, I. and Maruyama, S., Tactile Interfaces for Small Touch Screens. in Proceedings of UIST 2003, (Vancouver, Canada, 2003), ACM Press, 217-220.

16. Poupyrev, I., Maruyama, S. and Rekimoto, J., Ambient Touch: Designing tactile interfaces for handheld devices. In Proceedings of ACM UIST 2002, (Paris, France, 2002), ACM Press, 51-60.

17. Summers, I.R., Single Channel Information Transfer Through The Skin: Limitations and Possibilities. In Proceedings of ISAC 00, (2000). 